\title{
ARRANGEMENTS OF LINES WITH A LARGE NUMBER OF TRIANGLES
}

\author{
Z. FÜREDI AND I. PALÁSTI
}

\begin{abstract}
An arrangement of lines is constructed by choosing $n$ diagonals of the regular $2 n$-gon. This arrangement is proved to form at least $n(n-3) / 3$ triangular cells.
\end{abstract}

1. Introduction. We shall use the terminology of Grünbaum [5]. By an arrangement $\mathscr{A}$ of lines we mean a finite family of lines $L_{1}, \ldots, L_{n}$ in the real projective plane $\Pi$. The number of lines in $\mathscr{A}$ is denoted by $n(\mathscr{A})$. If no point belongs to more than two of these lines $L_{i}$, the arrangement is called simple. With an arrangement $\mathscr{A}$ there is associated a 2-dimensional cell-complex into which the lines of $\mathscr{A}$ decompose $\Pi$. It is well known that in a simple arrangement $\mathscr{A}$ the number of cells (or polygons) of that complex is $\left(n^{2}-n+2\right) / 2(n=n(\mathscr{A}))$. We shall denote by $p_{j}(\mathscr{A})$ the number of $j$-gons among the cells of (the complex associated with) $\mathscr{A}$.

2. Constructions. Let us denote by $P(O)$ a fixed point on the circle $\mathscr{C}$ with centre $C$. For any real $\alpha$, let $P(\alpha)$ be the point obtained by rotating $P(O)$ around $C$, with angle $\alpha$. Further denote by $L(\alpha)$ the straight line $P(\alpha) P(\pi-2 \alpha)$. In case $\alpha \equiv \pi-$ $2 \alpha(\bmod 2 \pi), L(\alpha)$ is the line tangent to $\mathscr{C}$ at $P(\alpha)$. (See Figure 1.)

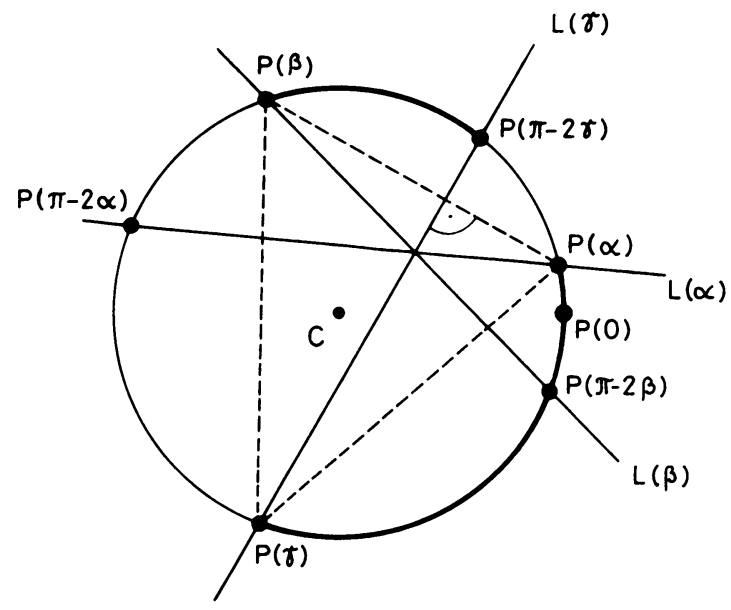

Figure 1

Received by the editors March 23, 1983 and, in revised form, January 19, 1984. 1980 Mathematics Subject Classification. Primary 51A20; Secondary 52A37.

(C)1984 American Mathematical Society $0002-9939 / 84 \$ 1.00+\$ .25$ per page 
562

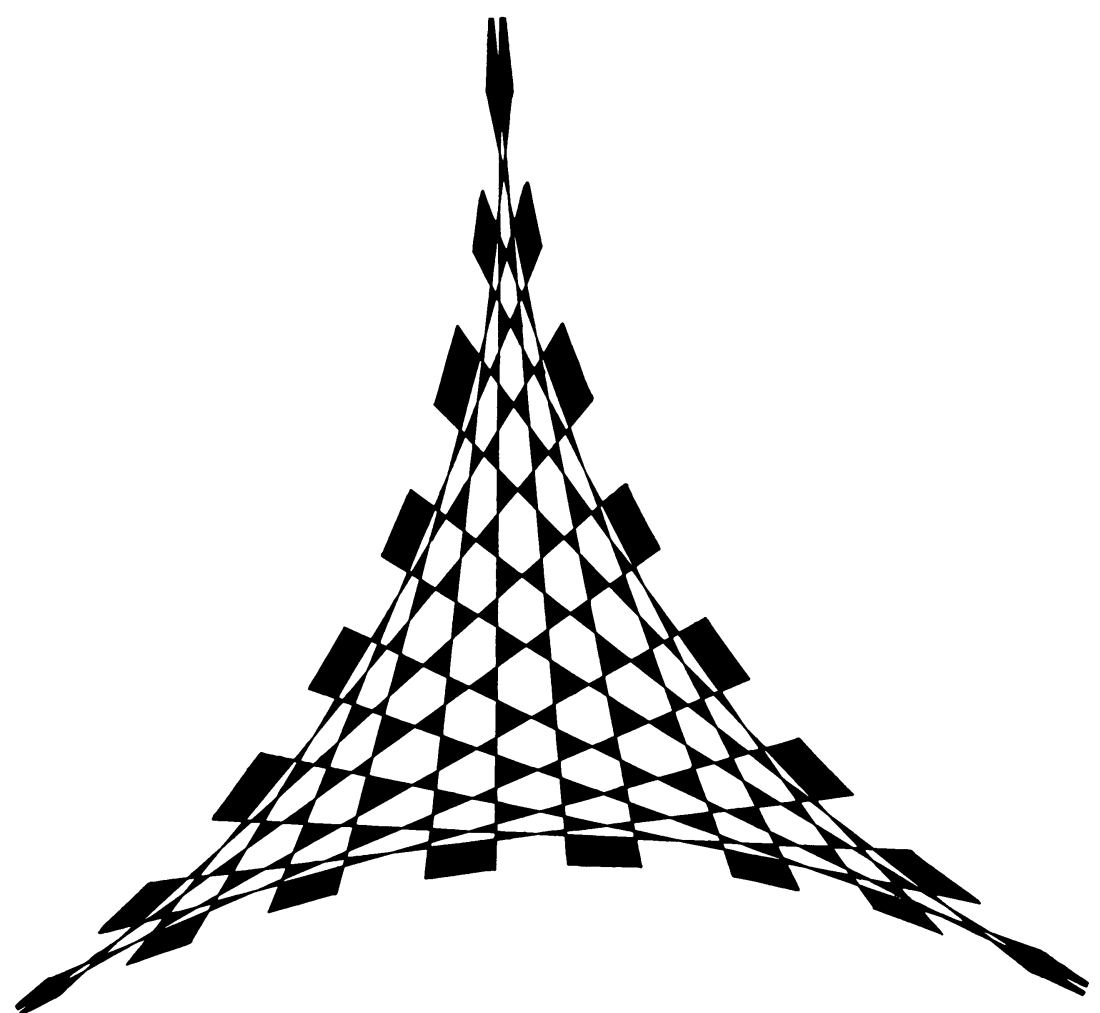

FIGURE 2

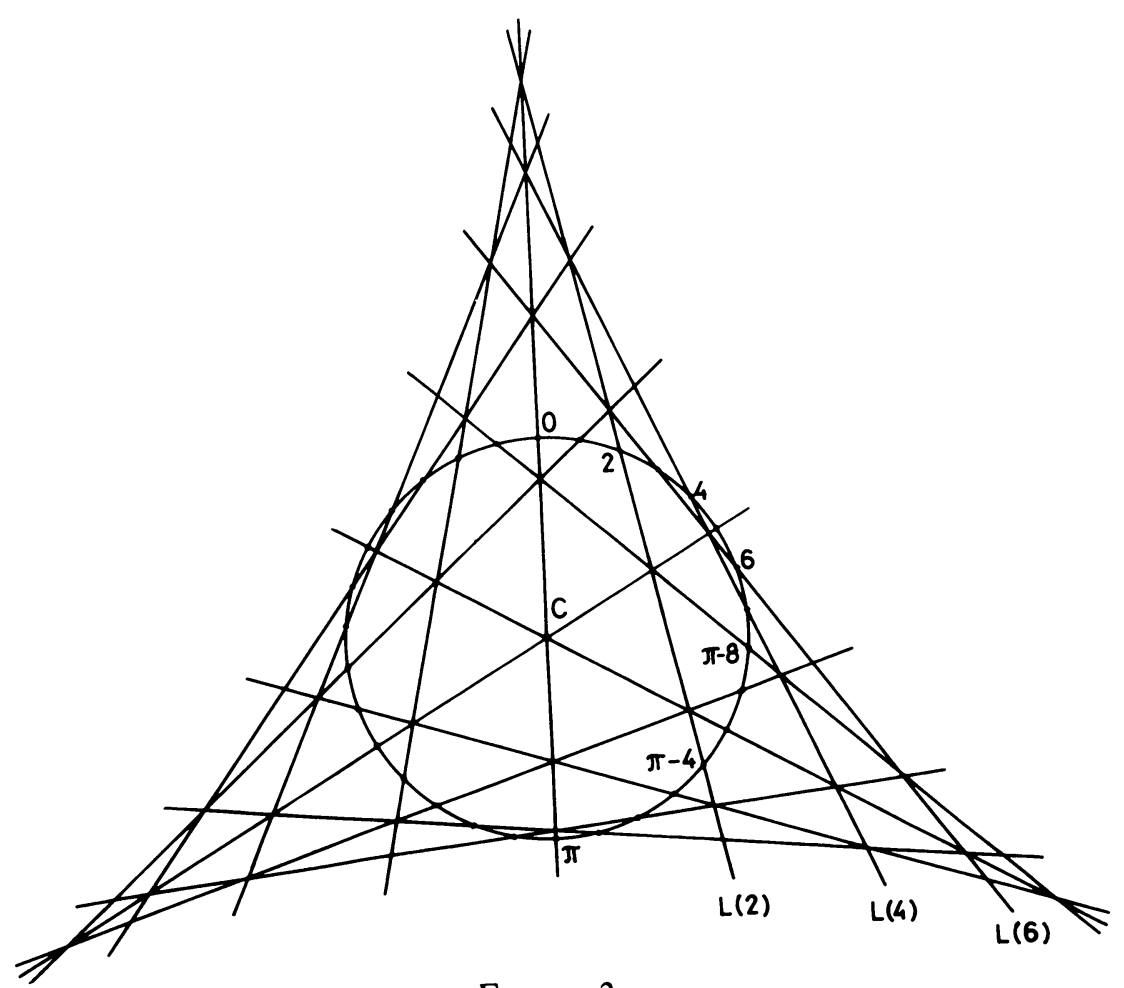

FIGURE 3 
EXAMPLE 1. Given any integer $n \geqslant 3$, put $\mathscr{A}_{n}=\{L((2 i+1) \pi / n): i=0,1, \ldots, n-$ 1). (See Figure 2.)

EXAMPle 2. Given any integer $n \geqslant 3$, put $\mathscr{B}_{n}=\{L(2 i \pi / n): i=0,1, \ldots, n-1\}$. (See Figure 3.)

Remark that our set of lines $\{L(\alpha): 0 \leqslant \alpha<2 \pi\}$ may be regarded as a set of tangents to the arcs of a hypocycloid of third order, drawn in a circle of centre $C$ and radius 3. The line $L(\alpha)$ is tangent to the arc of the cycloid at the $\alpha$ th point. (See Figure 4.) However, we shall not rely upon this fact in what follows.

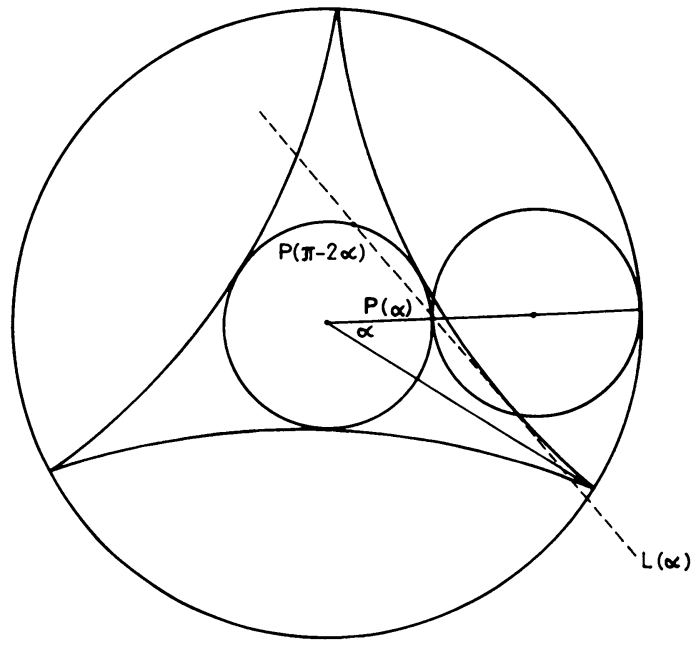

FIGURE 4

LEMMA. The lines $L(\alpha), L(\beta)$ and $L(\gamma)$ are concurrent if and only if $\alpha+\beta+\gamma \equiv 0$ $(\bmod 2 \pi)$.

Proof. If $\alpha+\beta+\gamma \equiv 0(\bmod 2 \pi)$, then the sum of lengths of (directed) arcs $(P(\alpha), P(\gamma))$ and $(P(\beta), P(\pi-2 \gamma))$ is equal to $\pi$. This implies that $L(\gamma)$ is perpendicular to the line $P(\alpha) P(\beta)$. Hence, the lines $L(\alpha), L(\beta)$ and $L(\gamma)$ are the altitudes of the triangle $P(\alpha) P(\beta) P(\gamma)$ (see Figure 1), consequently they meet at one point.

The reverse can be proved similarly.

3. Triangles in a simple arrangement. Grünbaum [5] (cf. Theorem 2.21) pointed out that the maximal number of triangles in a simple arrangement $p_{3}^{s}(n)=$ $\max \left\{p_{3}(\mathscr{A}): n(\mathscr{A})=n, \mathscr{A}\right.$ is simple $\}$ can be estimated by $p_{3}^{s}(n) \leqslant_{\llcorner} n(n-1) / 3$, for even $n$, and $p_{3}^{s}(n) \leqslant_{\llcorner} n(n-2) / 3$, if $n$ is odd. Moreover, he conjectured that this latter inequality holds for all $n, n \neq 1(\bmod 6)$. The exact value of $p_{3}^{s}(n)$ is known only for some small values of $n$. (Cf., e.g., Simmons $[15,16]$ for the case $n=15$, Grünbaum [5] for $n=20$ and Harborth [8] for $n=17$.) 
As far as we know, the best lower bound by now, $p_{3}^{s}(n)>(5 / 16+o(1)) n^{2}$, was due to Füredi and J. Pach (unpublished). We are now in a position to establish a sharper lower bound.

Property 1. $p_{3}\left(\mathscr{A}_{n}\right) \geqslant n(n-3) / 3$; hence $p_{3}^{s}(n)=n^{2} / 3+\mathcal{O}(n)$.

Proof. Let $\left(L_{i}, L_{j}\right)$ denote the intersection point of lines

$$
L_{i}=L((2 i+1) \pi / n) \text { and } L_{j}=L((2 j+1) \pi / n)
$$

Using the Lemma, we obtain that only $L((2 n-2 i-2 j-2) \pi / n)$ may cross $L_{i}$ and $L_{j}$ at $\left(L_{i}, L_{j}\right)$. But this line does not belong to $\mathscr{A}_{n}$, by definition. Therefore, the lines $L_{i}, L_{j}, L_{n-i-j-1}$ and $L_{i}, L_{j}, L_{n-i-j-2}$, resp., necessarily form triangular cells.

4. Quadrangles in a simple arrangement. Grünbaum conjectured that $p_{4}(\mathscr{A}) \geqslant 1$ for any simple arrangement with $n(\mathscr{A})>16$. (See 2.12 in [5], cf. [15, 16].) As far as we know one cannot find in the literature any example of a simple arrangement containing $\leqslant o\left(n^{2}\right)$ quadrangles. In view of this, Grünbaum's conjecture is surprisingly modest. It is easy to prove that

PROPERTY 2. The simple arrangement $\mathscr{A}_{n}$ contains only 3 or 5 quadrangles for odd $n$, and $p_{4}\left(\mathscr{A}_{n}\right)=\mathcal{O}(n)$ is valid for all values of $n$.

The results of $\S \S 3$ and 4 are collected in Table 1 . (We remark that $p_{j}\left(\mathscr{A}_{n}\right)=0$ for $j \geqslant 7$.)

\section{TABLE 1}

\begin{tabular}{l|l|c|c|c}
$n \geqslant 5$ & $p_{3}\left(\mathscr{A}_{n}\right)$ & $p_{4}\left(\mathscr{A}_{n}\right)$ & $p_{5}\left(\mathscr{A}_{n}\right)$ & $p_{6}\left(\mathscr{A}_{n}\right)$ \\
\hline$n \equiv 0(\bmod 6)$ & $\frac{1}{3}\left(n^{2}-3 n\right)$ & $n / 2+6$ & $n-6$ & $\frac{1}{6}\left(n^{2}-6 n+6\right)$ \\
\hline$n \equiv \pm 1(\bmod 6)$ & $\frac{1}{3}\left(n^{2}-3 n+5\right)$ & 5 & $2 n-9$ & $\frac{1}{6}\left(n^{2}-9 n+20\right)$ \\
\hline$n \equiv \pm 2(\bmod 6)$ & $\frac{1}{3}\left(n^{2}-3 n+8\right)$ & $n / 2$ & $n-2$ & $\frac{1}{6}\left(n^{2}-6 n+2\right)$ \\
\hline$n \equiv 3(\bmod 6)$ & $\frac{1}{3}\left(n^{2}-3 n+9\right)$ & 3 & $2 n-9$ & $\frac{1}{6}\left(n^{2}-9 n+24\right)$ \\
\hline
\end{tabular}

5. Triangles in arbitrary arrangements. Grünbaum conjectures that for any arrangement $\mathscr{A}$ of $n$ lines, $p_{3}(\mathscr{A}) \leqslant n(n-1) / 3$ holds. (See [5].) Let $p_{3}(n)=$ $\max \left\{p_{3}(\mathscr{A}): n(\mathscr{A})=n\right\}$. The best upper bound was given by Purdy [11, 12], who proved that

$$
p_{3}(n) \leqslant \frac{7}{18} n(n-1)+\frac{1}{3} \text { for } n \geqslant 6
$$

The best lower bound, $p_{3}(n) \geqslant 4+n(n-3) / 3$, is due to Strommer [18]. His result uses a construction of Burr, Grünbaum and Sloane [1].

PROPERTY 3. $p_{3}\left(\mathscr{B}_{n}\right) \geqslant 4+n(n-3) / 3$.

More exactly $p_{3}\left(\mathscr{B}_{n}\right)=n(n-3) / 3+6-2 \varepsilon / 3$, where $\varepsilon=0,2,2$ according to whether $n \equiv 0,1,2(\bmod 3)$. Further, $p_{4}\left(\mathscr{B}_{n}\right)=n-6+\varepsilon$, and $p_{j}\left(\mathscr{B}_{n}\right)=0$ for $j \geqslant 5$. The proof is easy.

6. The orchard problem. Given a vertex $V$ in an arrangement $\mathscr{A}$, denote by $t(V, \mathscr{A})$ the multiplicity of $V$, i.e. the number of lines of $\mathscr{A}$ incident to $V$. Further, let $t_{j}(\mathscr{A})$ denote the number of vertices of multiplicity $j(2 \leqslant j \leqslant n)$. We use the 
notation $t_{j}(n)=\max \left\{t_{j}(\mathscr{A}): n(\mathscr{A})=n\right\}$. The "orchard problem" has been investigated for about 150 years. It can be formulated as follows: find the value of $t_{3}(n)$. Significant progress has been made by Burr, Grünbaum and Sloane [1]. They proved that $t_{3}(n) \geqslant 1+\llcorner n(n-3) / 6$, by construction using elliptic integrals. Moreover, they conjectured that this result is sharp if $n$ is large enough. (Confer [1] for a complete (historical) bibliography of the subject.) Our construction (see Example 2) is much simpler, but this is only a special case of their idea. (The equation of the poles of $L(\alpha)$ 's is $\left(x^{2}+y^{2}\right)(3 x-1)=4 x^{3}$. This can be transformed into the form $y^{2}=4 x^{3}-(1 / 12) x-(1 / 216)$. Cf. [1].)

Property 4. $t_{2}\left(\mathscr{B}_{n}\right)=n-3+\varepsilon, t_{3}\left(\mathscr{B}_{n}\right)=1+{ }_{\llcorner} n(n-3) / 6$, and $t_{j}\left(\mathscr{B}_{n}\right)=0$ for $j \geqslant 4$.

It should be noted that recently Szemeredi and Trotter [19] proved that there exist $c$ and $c^{\prime}$ positive real numbers such that $c n^{2} / k^{3}<\max _{\mathscr{A}} \sum_{i \geqslant k} t_{i}(\mathscr{A})<c^{\prime} n^{2} / k^{3}$ for all $n>k^{2}$.

7. Two-coloring of arrangements. It is easy to prove by induction that the cells of a (not necessarily simple) arrangement $\mathscr{A}$ in the Euclidean plane can be colored by two colors (e.g., black and white) so that any two regions with a common side get different colors. Let $b=b(\mathscr{A})$ and $w=w(\mathscr{A})$ denote the numbers of black and white polygons. Without loss of generality we can assume that $b \geqslant w$. L. Fejes Tóth [3] raised the following question: What is the maximum of the ratio $b / w$ ? Palásti [10] proved that $b / w<2$ for $n(\mathscr{A}) \leqslant 9$. Upper bounds were given by Grünbaum [6], Simmons and Wetzel [17] and for higher dimensions by Purdy and Wetzel [13]. However, the exact value of $\max b / w$ is known only for some small values of $n$ with $n \leqslant 16$.

Grünbaum proved that $b \leqslant 2 w-2$ for all arrangements $\mathscr{A}$ with $n(\mathscr{A}) \geqslant 3$. For $n=3,5,9$ and 15 equality holds. Our Example 1 shows

PROPERTY 5. The number of black regions $b\left(\mathscr{A}_{n}\right)=\left(n^{2}+\varepsilon\right) / 3$, where $\varepsilon=0,2,2$ if $n \equiv 0,1,2(\bmod 3)$. So we get $b\left(\mathscr{A}_{n}\right)=2 w\left(\mathscr{A}_{n}\right)-(n+2-\varepsilon)$.

8. Gallai points. Let $\mathscr{A}$ be an arrangement of $n$ lines of the projective plane such that it does not contain a common point (i.e., $t_{n}(\mathscr{A})=0$ ). T. Gallai [4] proved that in this case there exist two lines from $\mathscr{A}$ whose intersection point has multiplicity 2 . This statement was improved by Kelly and Moser [9] $\left(t_{2}(\mathscr{A}) \geqslant 3 n / 7\right)$ and recently by $S$. Hansen [7] $\left(t_{2}(\mathscr{A}) \geqslant\left\llcorner n / 2_{\lrcorner}\right)\right.$.

The following question was posed by P. Erdős [2]. Let us suppose that the arrangement $\mathscr{A}$ does not contain a point with multiplicity more than 3 . Then does there exist a Gallai triangle, i.e. three lines from $\mathscr{A}$ such that their three intersection points have multiplicity 2 , or not? Our construction $\mathscr{B}_{n}$ shows that the answer is negative for $n \geqslant 4, n \neq 0(\bmod 9)$. Another problem on Gallai points can be found in [2].

9. Acknowledgements. The authors are indebted to J. Pach for his helpful suggestions. 


\section{REFERENCES}

1. S. Burr, B. Grünbaum and N. J. A. Sloane, The orchard problem, Geom. Dedicata 2 (1974), 397-424.

2. P. Erdős, Combinatorial problems in geometry, Math. Chronical 12 (1983), 35-54.

3. L. Fejes Tóth, A combinatorial problem concerning oriented lines in the plane, Amer. Math. Monthly 82 (1975), 387-389.

4. T. Gallai, See H. S. M. Coxeter: Introduction to geometry, Wiley, New York and London, 1961.

5. B. Grünbaum, Arrangements and spreads, Amer. Math. Soc., Providence, R.I., 1972.

6. __ Two-coloring the faces of arrangements, Period. Math. Hungar. 11 (1980), 181-185.

7. S. Hansen, Contributions to the Sylvester-Gallai-theory, $\mathrm{Ph} . \mathrm{D}$. Thesis, Copenhagen, 1981.

8. H. Harborth, Two-colorings of simple arrangements, Colloq. Math. Soc. Janos Bolyai, Eger, 1981 (to appear).

9. L. M. Kelly and W. O. J. Moser, On the number of ordinary lines determined by $n$ points, Canad. J. Math. 10 (1958), 210-219.

10. I. Palásti, The ratio of black and white polygons of a map generated by general straight lines, Period. Math. Hungar. 7 (1976), 91-94.

11. G. B. Purdy, Triangles in arrangements of lines, Discrete Math. 25 (1979), 157-163.

12. Triangles in arrangement of lines. II, Proc. Amer. Math. Soc. 79 (1980), 77-81.

13. G. B. Purdy and J. E. Wetzel, Two-coloring inequalities for Euclidean arrangements in general position, Discrete Math. 31 (1980), 53-58.

14. S. Roberts, On the figures formed by the intercepts of a system of straight lines in a plane, and on analogous relations in space of three dimensions, Proc. London Math. Soc. 19 (1887-1888), 405-422.

15. G. J. Simmons, A quadrilateral-free arrangement of sixteen lines, Proc. Amer. Math. Soc. 34 (1972), 317-318.

16. __ A maximal 2-arrangement of sixteen lines in the projective plane, Period. Math. Hungar. 4 (1973), 21-23.

17. G. J. Simmons and J. E. Wetzel, A two-coloring inequality for Euclidean two-arrangements, Proc. Amer. Math. Soc. 77 (1979), 124-127.

18. T. O. Strommer, Triangles in arrangements of lines, J. Combin. Theory Ser. A 23 (1977), 314-320.

19. E. Szemerédi and W. T. Trotter, Extremal problems in discrete geometry, Combinatorica 3 (1983).

Mathematics Institute, Hungarian Academy of Science, Budapest H - 1364 P. O. Box 127, HUNGARY 